\title{
Gini coefficients for measuring the distribution of sexually transmitted infections among individuals with different levels of sexual activity
}

\author{
Sandro Gsteiger ${ }^{1}$, Nicola Low ${ }^{1}$, Pam Sonnenberg ${ }^{2}$, Catherine H Mercer $^{2}$, Christian L Althaus ${ }^{\text {Corresp. } 1}$ \\ 1 Institute of Social and Preventive Medicine (ISPM), University of Bern, Bern, Switzerland \\ 2 Institute for Global Health, University College London, London, United Kingdom \\ Corresponding Author: Christian L Althaus \\ Email address: christian.althaus@alumni.ethz.ch
}

Objectives. Gini coefficients have been used to describe the distribution of Chlamydia trachomatis (CT) infections among individuals with different levels of sexual activity. The objectives of this study were to investigate Gini coefficients for different sexually transmitted infections (STIs), and to determine how STI control interventions might affect the Gini coefficient over time.

Methods. We used population-based data for sexually experienced women from two British National Surveys of Sexual Attitudes and Lifestyles (Natsal-2: 1999-2001; Natsal-3: 2010-2012) to calculate Gini coefficients for CT, Mycoplasma genitalium (MG), and human papillomavirus (HPV) types 6, 11, 16 and 18. We applied bootstrap methods to assess uncertainty and to compare Gini coefficients for different STIs. We then used a mathematical model of STI transmission to study how control interventions affect Gini coefficients.

Results. Gini coefficients for CT and MG were 0.33 (95\% confidence interval (Cl): 0.18-0.49) and 0.16 (95\% Cl: 0.02-0.36), respectively. The relatively small coefficient for MG suggests a longer infectious duration compared with CT. The coefficients for HPV types 6, 11, 16 and 18 ranged from 0.15-0.38. During the decade between Natsal-2 and Natsal-3, the Gini coefficient for CT did not change. The transmission model shows that higher STI treatment rates are expected to reduce prevalence and increase the Gini coefficient of STIs. In contrast, increased condom use reduces STI prevalence but does not affect the Gini coefficient.

Conclusions. Gini coefficients for STIs can help us to understand the distribution of STIs in the population, according to level of sexual activity, and could be used to inform STI prevention and treatment strategies. 
1 Gini coefficients for measuring the distribution of sexually transmitted infections among 2 individuals with different levels of sexual activity 3

4 Sandro Gsteiger ${ }^{1}$, Nicola Low ${ }^{1}$, Pam Sonnenberg ${ }^{2}$, Catherine H. Mercer ${ }^{2}$, Christian L. Althaus ${ }^{1}$ 5

$6{ }^{1}$ Institute of Social and Preventive Medicine (ISPM), University of Bern, Bern, Switzerland 7 2Institute for Global Health, University College London, London, UK

8

9 Corresponding author: Christian L. Althaus, Institute of Social and Preventive Medicine (ISPM), 10 University of Bern, Mittelstrasse 43, 3012 Bern, Switzerland; christian.althaus@alumni.ethz.ch. 


\section{Abstract}

12

13 Objectives. Gini coefficients have been used to describe the distribution of Chlamydia 14 trachomatis (CT) infections among individuals with different levels of sexual activity. The 15 objectives of this study were to investigate Gini coefficients for different sexually transmitted 16 infections (STIs), and to determine how STI control interventions might affect the Gini 17 coefficient over time.

19 Methods. We used population-based data for sexually experienced women from two British

20 National Surveys of Sexual Attitudes and Lifestyles (Natsal-2: 1999-2001; Natsal-3: 2010-2012)

21 to calculate Gini coefficients for CT, Mycoplasma genitalium (MG), and human papillomavirus 22 (HPV) types 6, 11, 16 and 18. We applied bootstrap methods to assess uncertainty and to 23 compare Gini coefficients for different STIs. We then used a conceptual transmission model for 24 STIs to study how control interventions affect Gini coefficients.

25

26 Results. Gini coefficients for CT and MG were 0.33 (95\% confidence interval (CI): 0.18-0.49) 27 and $0.16(95 \%$ CI: 0.02-0.36), respectively. The relatively small coefficient for MG suggests a 28 longer infectious duration compared with CT. The coefficients for HPV types 6, 11, 16 and 18 29 ranged from 0.15-0.38. During the decade between Natsal-2 and Natsal-3, the Gini coefficient for CT did not change. The transmission model shows that higher STI treatment rates are expected to reduce prevalence and increase the Gini coefficient of STIs. In contrast, increased condom use reduces STI prevalence but does not affect the Gini coefficient.

34 Conclusions. Gini coefficients for STIs can help us to understand the distribution of STIs in the 35 population, according to level of sexual activity, and could be used to inform STI prevention and treatment strategies. 


\section{Introduction}

38

39 Understanding how sexually transmitted infections (STIs) are distributed among individuals is 40 important both from a biological and from a public health perspective. Differences in STI 41 prevalence within a population, between groups with varying levels of sexual activity, can 42 provide information about biological and epidemiological characteristics of the infection. For 43 example, an STI with a long infectious duration, such as human papillomavirus (HPV), will tend 44 to be spread more evenly across a population than an STI with a short infectious duration, such 45 46 as Neisseria gonorrhoeae (NG). This observation can be explained by the fact that STIs with short infectious durations require a higher rate of sexual partner change for sustained spread in the population. NG is thus more concentrated in a small subgroup of individuals with high sexual activity. Such ideas were initiated in the late 1970s and led to the concept of the 'core group' [1]. In 1990, Brunham \& Plummer [2] inferred the size of core groups for various STIs from the biological parameters that describe transmissibility and infectious duration, and discussed the implications for selecting adequate STI control strategies.

52

53 The Gini coefficient can be used to quantify the degree of concentration of an STI in a 54 population. Originally introduced for describing inequalities in income distributions [3], the Gini coefficient provides a general tool to measure the distribution or imbalance of a disease outcome in relation to an exposure variable or risk factor [4], such as the geographic location or sexual behavior. A Gini coefficient of zero denotes perfect equality where an infection is equally distributed across a population. For infections that are concentrated in specific subpopulations, the Gini coefficient can increase up to a maximal value of one. The Lorenz curve is a visual representation of the cumulative distribution of a disease when ordered according to the risk factor [5]. The diagonal line on a Lorenz curve plot denotes perfect equality, e.g., every subpopulation has the same prevalence of an STI.

63

64 Several groups have used Gini coefficients and Lorenz curves to describe how Chlamydia 65 trachomatis (CT), NG, syphilis or herpes are distributed across different geographical regions in

66 Canada [6], the UK [7] and the US [8-10]. These findings have helped to summarize inequalities 67 in STI distributions, assess the suitability of geographically targeted interventions, and provide 
68 insights into the epidemic phases of the STIs over time. Chen et al. [11] were the first to apply

69 the concept of Gini coefficients in mathematical transmission models. They proposed a

70 metapopulation modeling framework that better captures the sociogeographic epidemiology of

71 NG and compared the resulting Gini coefficients with empirical estimates.

72

73 The way in which modifiable factors, such as sexual activity and STI control interventions affect

74 the Gini coefficient has been less-well studied. Previously, we described the distribution of CT

75 infections among individuals with different sexual activity using Lorenz curves and Gini

76 coefficients to calibrate dynamic transmission models [12]. We used data from the second British

77 Survey of Sexual Attitudes and Lifestyles (Natsal-2, 1999-2001) [13], which included CT test

78 results (samples were later tested for HPV [14]). The most recent survey (Natsal-3, 2010-2012)

79 also provides information on HPV and Mycoplasma genitalium (MG) positivity and offers a

80 unique opportunity to study relationships between sexual behavior and STI prevalence [15-17].

81

82 This study had two objectives. First, we wanted to estimate and compare the Gini coefficients for

83 different STIs and over time using data from two Natsal surveys. Second, we used a

84 mathematical transmission model to obtain general insights into how Gini coefficients are

85 expected to change as a result of STI control interventions.

86

87 Methods

88

89 Data

90 Natsal-3 is a population-based probability sample survey of sexual attitudes and lifestyles

91 conducted in Britain (England, Scotland, and Wales) and carried out from 2010 to 2012 [18,19].

92 The sample consists of 15162 women and men aged 16-74 years. A subsample of participants

93 aged 16-44 years who reported at least one sexual partner over their lifetime were asked to

94 provide urine samples, resulting in laboratory confirmed STI test results from 2665 women and

951885 men [15]. Urine was tested for the presence of CT, MG, type-specific HPV, NG, and HIV

96 antibodies. To compare Gini coefficients for CT over time, we also used data from the Natsal-2

97 survey, which was carried out in 1999-2001 [20]. This survey includes 11,161 women and men

98 aged 16-44 years. Urine samples for ligase chain reaction (LCR) testing for CT were available 
99 for a subset of 2055 and 1474 sexually active women and men aged 18-44 years [13]. Unless

100 otherwise stated, we used the subpopulations that provided urine samples for our analysis from

101 both surveys. Sexual behavior and urine sample data were individually weighted to adjust for

102 unequal selection probabilities and to correct for the age, gender and regional profiles in the

103 survey sample. For simplicity, we did not include same-sex contacts for the sexual behavior

104 variables because only $2.2 \%$ of the population reported a new same-sex partner during time

105 period covered by Natsal-3. The full datasets of both surveys are available from the UK Data

106 Archive at the University of Essex (http://data-archive.ac.uk).

107

108

Statistical analysis

109 We used Lorenz curves to plot the cumulative proportion of specific STI infections $\left(y_{i}\right)$ as a

110 function of the cumulative proportion of the population $\left(x_{i}\right)$, after population sub-groups $i$ (

$111 i=1,2, \ldots, n)$ have been ranked according to their level of sexual activity. The Gini coefficient is

112 defined as the area between the line of equality and the Lorenz curve over the total area below

113 the line of equality:

114

$$
G=1-\sum_{i=1}^{n}\left(x_{i}-x_{i-1}\right)\left(y_{i}+y_{i-1}\right),
$$

115 where $x_{0}=y_{0}=0$ and $x_{n}=y_{n}=1$.

116

117 We derived Lorenz curves and estimated Gini coefficients for CT, MG, and HPV types 6, 11, 16

118 and 18. We focused on these four HPV genotypes because they are present in the widely used

119 quadrivalent vaccine and are frequently considered in dynamic transmission models [21]. We did

120 not include NG and HIV in our analysis because of the small numbers of positive tests. We used

121 the number of new opposite-sex partners in the last year as the exposure variable summarizing

122 sexual activity because of its strong association with STI prevalence [12], and frequent use to

123 parameterize dynamic transmission models [22]. Owing to the larger sample size for female

124 respondents and a potential bias resulting from lower test sensitivity to detect HPV infections in

125 male urine [15], we focused our analysis on women and provide a separate analysis for men as

126 Supplemental Information. We constructed bootstrap confidence intervals (CIs) for the Gini

127 coefficients and point-wise bootstrap confidence bands for the Lorenz curves by sampling with 
128 replacement $[8-10,23]$. We calculated the 2.5 th and 97.5 th percentiles from 1000 bootstrapped

129 Gini coefficients and Lorenz curves.

130

131 Transmission model

132 We adapted a previously described mathematical model of STI transmission [24] to investigate

133 how changes in infectious duration and transmissibility affect the prevalence and the Gini

134 coefficient of an STI in a simulated population. We stratified the population according to sexual

135 activity [1,25], i.e., we assumed $n$ different sexual activity classes with $0,1,2, \ldots, n$-1 new

136 opposite-sex partners per year. For simplicity, we assumed that sexual activity and the natural

137 history and transmission of the infection are the same in women and men. The SIS (susceptible-

138 infected-susceptible) transmission dynamics can be described by the following ordinary

139 differential equation (ODE):

140

$$
\frac{d y_{i}}{d t}=\mu \sum_{j=1}^{n} x_{j} y_{j}+\beta c_{i}\left(1-y_{i}\right) \sum_{j=1}^{n} \rho_{i j} y_{j}-\gamma y_{i}-\mu y_{i},
$$

141

where $y_{i}$ is the proportion of individuals in sexual activity class $i$ who are infected, and $x_{i}$ is the

142 proportion of all individuals who belong to sexual activity class $i$. The first and last term of the

143 equation describe how individuals can change their sexual activity class at rate $\mu$ and be

144 redistributed to either the same or another sexual activity class proportional to the size of sexual

145 activity classes $[24,26]$. The middle terms describe the process of transmission and the clearance

146 of infection at rate $\gamma$. Susceptible individuals $1-y_{i}$ have an average of $c_{i}$ new opposite-sex

147 partners per year. $\beta$ is the per partnership transmission probability and $y_{j}$ is the probability that a

148 partner in sexual activity class $j$ is infected. $\rho_{i j}$ represents the elements of the sexual mixing

149 matrix [27]

150

$$
\rho_{i j}=\epsilon \delta_{i j}+(1-\epsilon) \frac{c_{j} x_{j}}{\sum_{l=1}^{n} c_{l} x_{l}},
$$

151 where $\delta_{i j}$ denotes the Kronecker delta (it is equal to 1 if $i=j$ and to 0 otherwise).

152

153 The proportion of individuals in sexual activity classes $x_{i}$ with $c_{i} \in 0,1,2, \ldots, n-1$ new opposite-

154 sex partners per year were based on Natsal-2. All data for 18-44 year old women and men were 
155 pooled and weighted. Since the transmission model is primarily used for illustrative purposes, we

156 did not include changes in sexual behavior, between Natsal-2 and Natsal-3, which were small

157 [19]. We set $\mu=1$ per year as described previously [24,26], and set $\epsilon=0$, i.e., we assumed

158 random proportional mixing between different sexual activity classes [27]. We then chose a

159 particular combination of the infectious duration $(1 / \gamma)$ and the per partnership transmission

160 probability $(\beta)$ and ran the model into endemic equilibrium. We recorded the simulated STI

161 prevalence and computed the Gini coefficient as described above, i.e., by calculating the

162 cumulative proportion of infections as a function of the cumulative proportion of the population,

163 ranked by sexual activity $i$. We repeated this process for various combinations of the infectious

164 duration and the per partnership transmission probability, which allowed us to map between the

165 (unobservable) model parameters and the simulated (observable) summary measures prevalence

166 and Gini coefficient.

167

168 We further used the transmission model to investigate how two hypothetical CT control

169 interventions affect infection prevalence and the Gini coefficient. First, we calibrated the

170 infectious duration and the per partnership transmission probability such that the simulated CT

171 prevalence and Gini coefficient correspond to the estimates from Natsal- $2(1 / \gamma=1.75$ years, $\beta=$

$17219 \%$ ). Second, we simulated the expected changes in prevalence and the Gini coefficient as a

173 result of a) an increase in CT screening coverage aiming to detect asymptomatic CT cases, and

174 b) the effects of an educational campaign that leads to a change in sexual behavior and/or an

175 increase in condom use. We assumed that the first intervention would reduce the overall

176 infectious duration by $10 \%$. For the second intervention, we assumed a $10 \%$ reduction in the

177 product of the per partnership transmission probability $(\beta)$ and the number of opposite-sex

178 partners $\left(c_{i}\right)$. Note that these hypothetical interventions do not necessarily predict the quantitative

179 effects of real-world interventions; instead, they provide a qualitative picture of how prevalence

180 and Gini coefficients are expected to change.

181

182 Data analyses and model simulations were performed in the R software environment for

183 statistical computing [28]. All code files are available on GitHub

184 (https://github.com/calthaus/gini). 


\section{Results}

189

190

Lorenz curves and Gini coefficients

191 The Lorenz curves for MG and HPV types 6, 11 and 16 are closer to the diagonal line than the 192 Lorenz curves for CT and HPV 18 (Fig. 1A). This indicates that CT and HPV-18 in women are 193 more strongly associated with the number of new opposite-sex partners in the last year than MG 194 and the other type-specific HPV. The Gini coefficients mirror this observation and are higher for 195 CT (0.33) and HPV 18 (0.38) than for MG and the different HPV types $(\leq 0.22)$ (Table 1).

196 However, the bootstrapped confidence intervals for the Lorenz curves are wide (figure 1B), 197 resulting in considerable uncertainty in the estimated Gini coefficients. The Lorenz curves for 198 CT for the two survey periods of Natsal-2 (1999-2001) and Natsal-3 (2010-2012) are similar 199 (figure 1C) with a Gini coefficient in Natsal-2 of 0.30 (95\% CI: 0.12-0.50) and in Natsal-3 of $200 \quad 0.33$ (95\% CI: 0.18-0.49).

201

202 [INSERT FIG. 1 HERE.]

203

204

[INSERT TABLE 1 HERE.]

205

206 We used the transmission model to perform a mapping between model parameters and the 207 simulated STI prevalence and Gini coefficient. Within the parameter ranges that are 208 representative for the considered STIs, we found a close to linear relationship between the Gini 209 coefficient, infection prevalence, infectious duration and per partnership transmissibility (Fig. 210 2A, dashed grid). STIs with a short infectious duration (e.g., 1 year) require frequent sexual 211 partner changes (i.e., a high number of opposite-sex partners) and are therefore characterized by 212 a high Gini coefficient. Longer infectious durations increase prevalence, facilitate STI 213 transmission between individuals with a low number of opposite-sex partners, and consequently 214 decrease the Gini coefficient. Interestingly, different values for the per partnership transmission 215 probability only influence prevalence but do not affect the Gini coefficient. These insights from 216 the transmission model potentially allow the inference of biological parameters for the different 
217 STIs in Natsal-3 (Fig. 2A, colored dots). Although the confidence intervals and the associated

218 uncertainty are large, CT and HPV 18 seem to be consistent with an infectious duration between

2191 and 2 years. MG and the other HPV types are consistent with longer infectious durations.

220

221 [INSERT FIG. 2 HERE.]

222

223

STI control interventions

224 We used the transmission model to examine how two hypothetical control interventions for CT 225 affect the Gini coefficient and infection prevalence. First, we assumed that the increase in CT 226 screening between the two survey periods of Natsal-2 (1999-2001) and Natsal-3 (2010-2012)

227 through the National Chlamydia Screening Programme (NCSP) in England [29] has resulted in a 228 reduction in the overall infectious duration. The transmission model predicts that this would 229 result in an increase of the Gini coefficient with a concurrent drop in prevalence (Fig. 2B). In 230 contrast, the point estimates of the Gini coefficient and prevalence both show a slight, albeit no 231 statistically significant increase between Natsal-2 and Natsal-3. The expected changes in 232 prevalence and Gini coefficient might be relatively small, however, and may remain within the $23395 \%$ CIs of the point estimates. Second, we simulated the effects of a decrease in the number of 234 opposite-sex partners and/or an increase in condom. Such a behavior change would be expected 235 to reduce the prevalence of CT without affecting the Gini coefficient.

236

237

\section{Discussion}

238

239

240

Building upon earlier work [12], we constructed Lorenz curves and estimated Gini coefficients in women to investigate how different STIs are distributed according to sexual activity. Gini

241 coefficients for CT and HPV 18 appear to be higher than for MG and HPV 6, 11 and 16. We

242 found no evidence that the Gini coefficient for CT changed between the two survey periods of

243 Natsal-2 and Natsal-3. Using a mathematical model of STI transmission, we found that a CT

244 screening intervention should reduce prevalence and increase the Gini coefficient, whilst condom 245 use reduced prevalence but did not affect the Gini coefficient.

247 A main strength of this study was the availability of Natsal-2 and Natsal-3, two very large data 
248 sets that measure both STI positivity and self-reported sexual behavior in probability samples of

249 the British general population. These comprehensive data sets allow comparison between

250 different STIs and over time. Calculating infection prevalence and Gini coefficients is

251 straightforward if suitable data are available. In contrast, obtaining biological parameters that

252 determine the transmission dynamics, such as the infectious duration or the transmission

253 probability, are notoriously difficult to obtain [30,31]. In this study, we used a mathematical

254 model of STI transmission with a detailed description of different sexual activity classes [24] to

255 explore the relationship between Gini coefficient, infection prevalence, infectious duration and

256 transmissibility.

257

258 There are several limitations to this study. First, despite the large overall sizes of the two Natsal

259 surveys, the relatively low prevalence of STIs in the general population [13-16] resulted in

260 relatively large uncertainties in the Lorenz curves and the corresponding Gini coefficients,

261 particularly for males (see Supplemental Information). Owing to the small sample sizes, we

262 pooled data over all age groups and restricted our analyses to the whole survey population.

263 Investigating sex- or age-specific differences in Gini coefficients would certainly be interesting

264 but is currently not feasible. Second, the limited sample size did not allow us to calculate Gini

265 coefficients for NG and HIV, which have a very low prevalence, and arguably high Gini

266 coefficient [15]. Third, the comparison of prevalence and Gini coefficients of CT between

267 Natsal-2 and Natsal-3 should also be treated with caution. The two surveys used different

268 laboratory tests and were not powered to detect changes in CT prevalence [15]. Fourth, we

269 defined the exposure variable as the number of new opposite-sex partners in the last year. Using

270 other exposure variables for our analysis would obviously affect the Lorenz curves and the Gini

271 coefficients, but would not necessarily be applicable for the mapping between model parameters

272 and the summary measures. Fifth, mathematical modeling necessarily involves several

273 assumptions and simplifications. As in the data analysis, we did not stratify the population

274 according to age, assumed the sexual behavior in women and men to be the same, and considered

275 the general population of those reporting sex with opposite-sex partners as a whole. Changes in

276 sexual behavior between Natsal-2 and Natsal-3 were minimal [19], so we did not take these into

277 account. We assumed fully proportional mixing which typically results in the best description of

278 infection prevalence in different sexual activity classes in models with a constant per partnership 
279 transmission probability [22,24,27]. We also did not consider sex-specific differences in the

280 infectious duration and the transmissibility of the various STIs, which might limit the application

281 of Gini coefficients for the inference of these parameters. Further, we assumed that individuals

282 who clear an infection can become reinfected, although CT and type-specific HPV infections

283 might confer temporal immunity [32,33]. Together, these modeling assumptions highlight that

284 the insights from our conceptual transmission model are primarily qualitative and that

285 quantitative results should be treated with caution.

286

287 The calculated Gini coefficients and prevalence of CT and HPV 18 in women in Natsal-3

288 suggest an infectious duration of 1-2 years, which is in good agreement with previous estimates

289 [30,34-36]. Our mapping indicates that the infectious durations for the other high-risk HPV type

29016 and low-risk HPV types 6 and 11 could be longer than 2 years. This interpretation would

291 contrast with previous studies that estimate similar infectious durations for HPV-16 and HPV-18

292 [34,35], and shorter infectious durations of less than a year for HPV 6 and 11 [34]. The

293 discrepancy could be a result of ignoring the effects of temporal immunity to reinfection. The

294 infectious duration for the other bacterial STI, MG, seems to be longer than for CT. There is

295 considerable uncertainty regarding the infectious duration of MG. One analysis, which used data

296 from a study of female students in London (UK), estimated the mean infectious duration at 15

297 months [37], which is in the same range as CT. The per partnership transmission probability is a

298 highly model-dependent parameter and depends on the type of sexual partnerships that are

299 considered. It is maybe not surprising that our mapping, which suggests relatively low per

300 partnership transmission probabilities of $10 \%$ to $25 \%$, is not consistent with estimates from other

301 modeling studies [31,33].

302

303 Our findings allowed us to interpret differences in Gini coefficients and changes over time for

304 different STIs, illustrating that Gini coefficients can serve beyond their original role as simple

305 statistical measures of exposure-outcome associations. We found that changes in the

306 transmission probability only influence infection prevalence. This means that while decreasing

307 the transmission probability (e.g., through increased condom use) decreases the overall burden of

308 an STI, it does not affect how an STI is distributed among individuals with different sexual

309 activity. Hence, the target groups for future control interventions should remain the same. In 
310 contrast, we showed that changes in the infectious duration (e.g., through increased testing and

311 treatment uptake) affects both the prevalence and Gini coefficient of an STI. A stronger

312 concentration of the infections among individuals with increased sexual activity would require a

313 change in the target groups for control interventions. A prerequisite for this newly proposed use

314 of Gini coefficients for STIs will be the availability of large data sets with a sufficiently high

315 infection prevalence. These could either be population-based surveys or smaller cohorts that

316 focus on individuals with an increased risk of acquiring STIs, such as men who have sex with

317 men (MSM).

318

319 In summary, our study illustrates that the Gini coefficient for measuring the distribution of an

320 STI among individuals with different sexual activity represents a simple proxy measure, which

321 combines epidemiological and behavioral data. Estimating Gini coefficients for the general

322 population or particular sub-populations, in combination with mathematical modeling, has the

323 potential to make inference of biological parameters that determine STI transmission and to

324 assess the impact of control measures. 
325 Acknowledgements Natsal-3 is a collaboration between University College London (UCL),

326 London School of Hygiene and Tropical Medicine (LSHTM), National Centre for Social

327 Research (NatCen), Public Health England (PHE), and the University of Manchester. Natsal-2

328 was a collaboration between UCL, LSHTM, NatCen, and the Health Protection Agency (now

329 PHE). We thank the study participants, the team of interviewers from NatCen Social Research,

330 and operations and computing staff from NatCen Social Research. Natsal-3 was approved by the

331 Oxfordshire Research Ethics Committee A (Ref: 10/H0604/27); Natsal-2 was approved by the

332 University College Hospital and North Thames Multi-Centre Research Ethics Committee and all

333 the Local Research Ethics Committees in Britain.

334 


\section{References}

336

3371 Hethcote Herbert W, Yorke JA. Gonorrhea transmission dynamics and control. Springer338 Verlag, Berlin 1984.

3392 Brunham RC, Plummer FA. A general model of sexually transmitted disease epidemiology $340 \quad$ and its implications for control. Med Clin North Am 1990;74:1339-52.

3413 Gini CW. Variability and Mutability, contribution to the study of statistical distributions and 342 relations. Studi Economico-Giuridici della R Universita de Cagliari 1912.

3434 Lee WC. Characterizing exposure-disease association in human populations using the 344 Lorenz curve and Gini index. Stat Med 1997;16:729-39.

3455 Lorenz MO. Methods of measuring the concentration of wealth. Journal of the American $346 \quad$ Statistical Association 1905;9:209-19.

3476 Elliott LJ, Blanchard JF, Beaudoin CM, Green CG, Nowicki DL, Matusko P, Moses S.

348 Geographical variations in the epidemiology of bacterial sexually transmitted infections in 349 Manitoba, Canada. Sex Transm Infect 2002;78 Suppl 1:1139-44.

3507 Monteiro EF, Lacey CJN, Merrick D. The interrelation of demographic and geospatial risk 351 factors between four common sexually transmitted diseases. Sex Transm Infect 2005;81:413526.

3538 Kerani RP, Handcock MS, Handsfield HH, Holmes KK. Comparative geographic 354 concentrations of 4 sexually transmitted infections. Am J Public Health 2005;95:324-30.

3559 Chesson HW, Sternberg M, Leichliter JS, Aral SO. Changes in the state-level distribution of 356 primary and secondary syphilis in the USA, 1985-2007. Sex Transm Infect 2010;86 Suppl 357 3:iii58-62.

35810 Chesson HW, Sternberg M, Leichliter JS, Aral SO. The distribution of chlamydia, 359 gonorrhoea and syphilis cases across states and counties in the USA, 2007. Sex Transm $360 \quad$ Infect 2010;86 Suppl 3:iii52-57.

36111 Chen MI, Ghani AC, Edmunds WJ. A metapopulation modeling framework for gonorrhoea 362 363 and other sexually transmitted infections in heterosexual populations. $J R$ Soc Interface

36412 Althaus CL, Turner KME, Schmid BV, Heijne JCM, Kretzschmar M, Low N. Transmission 365 of Chlamydia trachomatis through sexual partnerships: a comparison between three 
366 individual-based models and empirical data. J R Soc Interface 2012;9:136-46.

36713 Fenton KA, Korovessis C, Johnson AM, McCadden A, McManus S, Wellings K, Mercer

368 CH, Carder C, Copas AJ, Nanchahal K, Macdowall W, Ridgway G, Field J, Erens B. Sexual

369 behaviour in Britain: reported sexually transmitted infections and prevalent genital

370 Chlamydia trachomatis infection. Lancet 2001;358:1851-4. doi:10.1016/S0140-

371 6736(01)06886-6

37214 Johnson AM, Mercer CH, Beddows S, de Silva N, Desai S, Howell-Jones R, Carder C,

373 Sonnenberg P, Fenton KA, Lowndes C, Soldan K. Epidemiology of, and behavioural risk

374 factors for, sexually transmitted human papillomavirus infection in men and women in

375 Britain. Sex Transm Infect 2012;88:212-7. doi:10.1136/sextrans-2011-050306

37615 Sonnenberg P, Clifton S, Beddows S, Field, N, Soldan K, Tanton C, Mercer CH, da Silva

377 FC, Alexander S, Copas AJ, Phelps A, Erens B, Prah P, Macdowall W, Wellings K, Ison

378 CA, Johnson AM. Prevalence, risk factors, and uptake of interventions for sexually

379 transmitted infections in Britain: findings from the National Surveys of Sexual Attitudes and

380 Lifestyles (Natsal). Lancet 2013;382:1795-806. doi:10.1016/S0140-6736(13)61947-9

38116 Sonnenberg P, Ison CA, Clifton S, Field N, Tanton C, Soldan K, Beddows S, Alexander S,

382 Khanom R, Saunders P, Copas AJ, Wellings K, Mercer CH, Johnson AM. Epidemiology of

383 Mycoplasma genitaliumin British men and women aged 16-44 years: evidence from the

384 third National Survey of Sexual Attitudes and Lifestyles (Natsal-3). International Journal of

385 Epidemiology 2015;44:1982-94. doi:10.1093/ije/dyv194

38617 Tanton C, Mesher D, Beddows S, Soldan K, Clifton S, Panwar K, Field N, Mercer CH,

387 Johnson AM, Sonnenberg P. Human papillomavirus (HPV) in young women in Britain:

388 Population-based evidence of the effectiveness of the bivalent immunisation programme and

389 burden of quadrivalent and 9-valent vaccine types. Papillomavirus Res 2017;3:36-41.

$390 \quad$ doi:10.1016/j.pvr.2017.01.001

39118 Erens B, Phelps A, Clifton S, Mercer CH, Tanton C, Hussey D, Sonnenberg P, Macdowall

392 W, Field N, Datta J, Mitchell K, Copas AJ, Wellings K, Johnson AM. Methodology of the

393 third British National Survey of Sexual Attitudes and Lifestyles (Natsal-3). Sexually

394 Transmitted Infections 2013;sextrans-2013-051359. doi:10.1136/sextrans-2013-051359

39519 Mercer CH, Tanton C, Prah P, Erens B, Sonnenberg P, Clifton S, Macdowall W, Lewis R,

396 Field N, Datta J, Copas AJ, Phelps A, Wellings K, Johnson AM. Changes in sexual attitudes 
397

398

399

400

401

402

403

404

405

406

407

408

409

410

411

412

413

414

415

416

417

418

419

420

421

422

423

424

425

426

427

and lifestyles in Britain through the life course and over time: findings from the National Surveys of Sexual Attitudes and Lifestyles (Natsal). Lancet 2013;382:1781-94. doi:10.1016/S0140-6736(13)62035-8

20 Johnson AM, Mercer CH, Erens B, Copas AJ, McManus S, Wellings K, Fenton KA, Korovessis C, Macdowall W, Nanchahal K, Purdon S, Field J. Sexual behaviour in Britain: partnerships, practices, and HIV risk behaviours. Lancet 2001;358:1835-42. doi:10.1016/S0140-6736(01)06883-0

21 Brisson M, Bénard É, Drolet M, Bogaards JA, Baussano I, Vänskä S, Jit M, Boily MC, Smith MA, Berkhof J, Canfell K, Chesson HW, Burger EA, Choi YH, Freiesleben De Blasio B, De Vlas SJ, Guzzetta G, Hontelez JAC, Horn J, Jepsen MR, Kim JJ, Lazzarato F, Matthijsse SM, Mikolajczyk R, Pavelyev A, Pillsbury M, Shafer LA, Tully SP, Turner HC, Usher C, Walsh C. Population-level impact, herd immunity, and elimination after human papillomavirus vaccination: A systematic review and meta-analysis of predictions from transmission-dynamic models. The Lancet Public Health 2016;1:e8-e17.

22 Althaus CL, Heijne JC, Herzog SA, Roellin A, Low N. Individual and population level effects of partner notification for Chlamydia trachomatis. PLoS One 2012;7:e51438.

23 Davison AC, Hinkley DV. Bootstrap Methods and their Application. 1 edition. Cambridge; New York, NY, USA:: Cambridge University Press 1997.

24 Althaus CL, Choisy M, Alizon S. Number of sex acts matters for heterosexual transmission and control of Chlamydia trachomatis. PeerJ PrePrints 2015;3:e1164.

\section{doi:10.7287/peerj.preprints.940v1}

25 Garnett GP, Anderson RM. Factors controlling the spread of HIV in heterosexual communities in developing countries: patterns of mixing between different age and sexual activity classes. Philos Trans R Soc Lond B Biol Sci 1993;342:137-59.

26 Fingerhuth SM, Bonhoeffer S, Low N, Althaus CL. Antibiotic-Resistant Neisseria gonorrhoeae Spread Faster with More Treatment, Not More Sexual Partners. PLoS Pathog 2016;12:e1005611. doi:10.1371/journal.ppat.1005611

27 Garnett GP, Mertz KJ, Finelli L, Levine WC, St Louis ME. The transmission dynamics of gonorrhoea: modeling the reported behaviour of infected patients from Newark, New Jersey. Philos Trans R Soc Lond B Biol Sci 1999;354:787-97.

28 R Core Team. R: A Language and Environment for Statistical Computing. Vienna, Austria:: 
428

429

430

431

432

433

434

435

436

437

438

439

440

441

442

443

444

445

446

447

448

449

450

451

452

453

454

455

456

457

458

R Foundation for Statistical Computing 2016.

29 Chandra NL, Soldan K, Dangerfield C, Sile B, Duffell S, Talebi A, Choi YH, Hughes G, Woodhall SC. Filling in the gaps: estimating numbers of chlamydia tests and diagnoses by age group and sex before and during the implementation of the English National Screening Programme, 2000 to 2012. Euro Surveill 2017;22. doi:10.2807/1560-

\section{ES.2017.22.5.30453}

30 Althaus CL, Heijne JC, Roellin A, Althaus CL. Transmission dynamics of Chlamydia trachomatis affect the impact of screening programmes. Epidemics 2010;2:123-31.

31 Althaus CL, Heijne JCM, Low N. Towards More Robust Estimates of the Transmissibility of Chlamydia trachomatis. Sex Transm Dis 2012;39:402-4.

32 Brunham RC, Rey-Ladino J. Immunology of chlamydia infection: Implications for a chlamydia trachomatis vaccine. Nat Rev Immunol 2005;5:149-61. doi:10.1038/nri1551

33 Bogaards JA, Xiridou M, Coupé VMH, Meijer CJLM, Wallinga J, Berkhof J. Model-based estimation of viral transmissibility and infection-induced resistance from the age-dependent prevalence of infection for 14 high-risk types of human papillomavirus. Am J Epidemiol 2010;171:817-25. doi:10.1093/aje/kwp466

34 Insinga RP, Dasbach EJ, Elbasha, Liaw KL, Barr E. Incidence and duration of cervical human papillomavirus 6,11,16, and 18 infections in young women: An evaluation from multiple analytic perspectives. Cancer Epidemiol Biomarkers Prev 2007;16:709-15. doi:10.1158/1055-9965.EPI-06-0846

35 Johnson HC, Elfström KM, Edmunds WJ. Inference of type-specific HPV transmissibility, progression and clearance rates: a mathematical modeling approach. PLoS One 2012;7:e49614. doi:10.1371/journal.pone.0049614

36 Price MJ, Ades AE, Angelis DD, Welton NJ, Macleod J, Soldan K, Turner K, Simms I, Horner PJ. Mixture-of-exponentials models to explain heterogeneity in studies of the duration of Chlamydia trachomatis infection. Stat Med 2013;32:1547-60. doi:10.1002/sim.5603

37 Smieszek T, White PJ. Apparently-Different Clearance Rates from Cohort Studies of Mycoplasma genitalium Are Consistent after Accounting for Incidence of Infection, Recurrent Infection, and Study Design. PLoS One 2016;11:e0149087. 


\section{Table $\mathbf{1}$ (on next page)}

Estimated Gini coefficients for different sexually transmitted infections in women. 
1 Table 1: Estimated Gini coefficients for different sexually transmitted infections in women.

\begin{tabular}{|l|c|c|}
\hline Infection & Gini coefficient & 95\% confidence interval (CI) \\
\hline Chlamydia trachomatis & 0.33 & $0.18-0.49$ \\
\hline Mycoplasma genitalium & 0.16 & $0.02-0.36$ \\
\hline HPV 6 & 0.22 & $0.02-0.43$ \\
\hline HPV 11 & 0.17 & $0.04-0.31$ \\
\hline HPV 16 & 0.15 & $0.04-0.27$ \\
\hline HPV 18 & 0.38 & $0.10-0.64$ \\
\hline
\end{tabular}

2 


\section{Figure 1}

Lorenz curves representing the cumulative proportion of STI infections in women as a function of the cumulative proportion of the population, after population sub-groups have been ranked by the number of new opposite-sex partners in the last year.

(A) Lorenz curves for different STIs. Data: Natsal-3. (B) Uncertainty around Lorenz curve for CT. The blue areas represent point-wise 50\% (dark blue) and 95\% (light blue) confidence bands. Data: Natsal-3. (C) Comparison of Lorenz curves for CT between Natsal-2 (dashed line) and Natsal-3 (solid line). In all graphs, the diagonal line (black dotted line) denotes perfect equality, i.e., an equal dispersion of the infection across population sub-groups. 


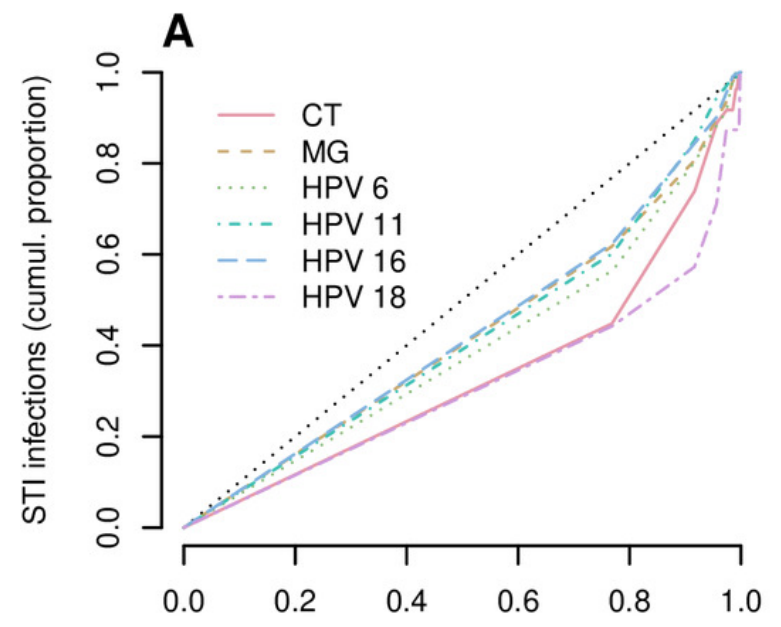

New opposite-sex partners last year (cumul. proportion)

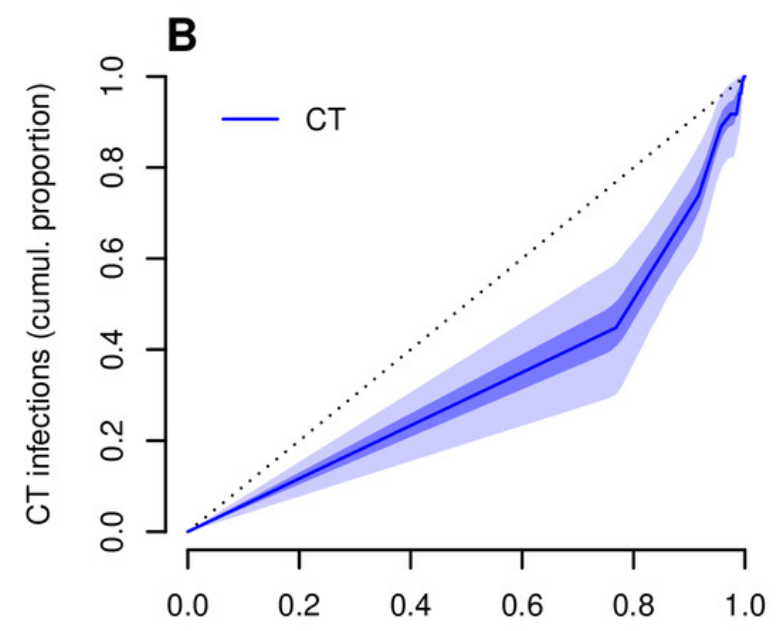

New opposite-sex partners last year (cumul. proportion)

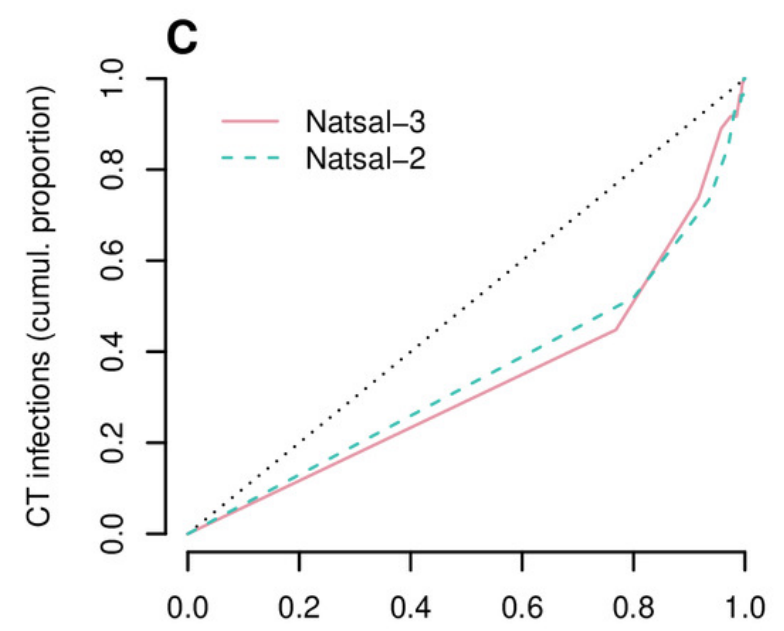

New opposite-sex partners last year (cumul. proportion)

Peer) reviewing PDF | (2019:08:40173:1:1:NEW 20 Nov 2019) 


\section{Figure 2}

Relationship between Gini coefficient, STI prevalence, infectious duration and transmissibility.

(A) Gini coefficients and STI prevalence for women in Natsal-3 (colored dots). Modelled values for different combinations of the infectious duration and the per partnership transmission probability are projected on the graph (dashed grid). (B) Expected impact of control measures on Gini coefficients and prevalence of female CT between Natsal-2 and Natsal-3. The black arrows denote a $10 \%$ reduction in the per partnership transmission probability (horizontal arrows) or the infectious duration (diagonal arrows).
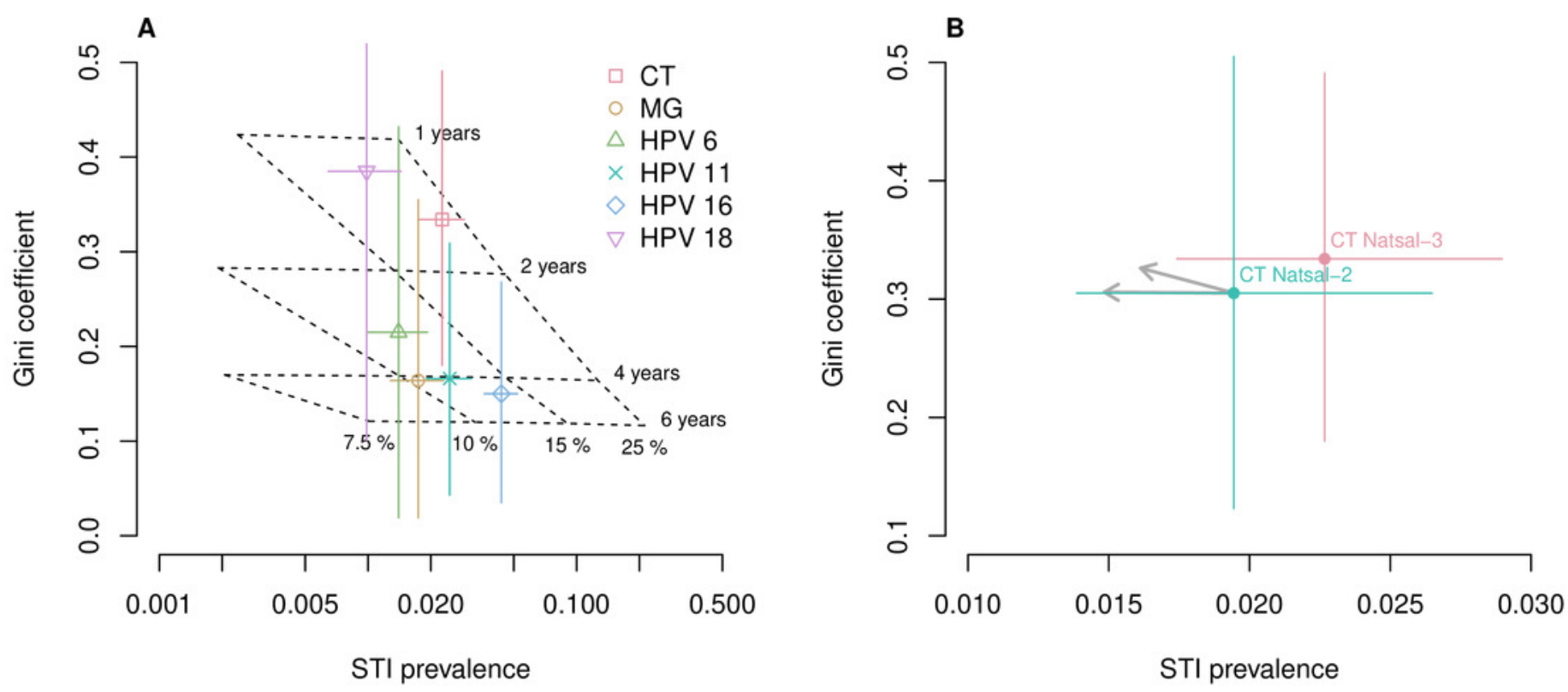